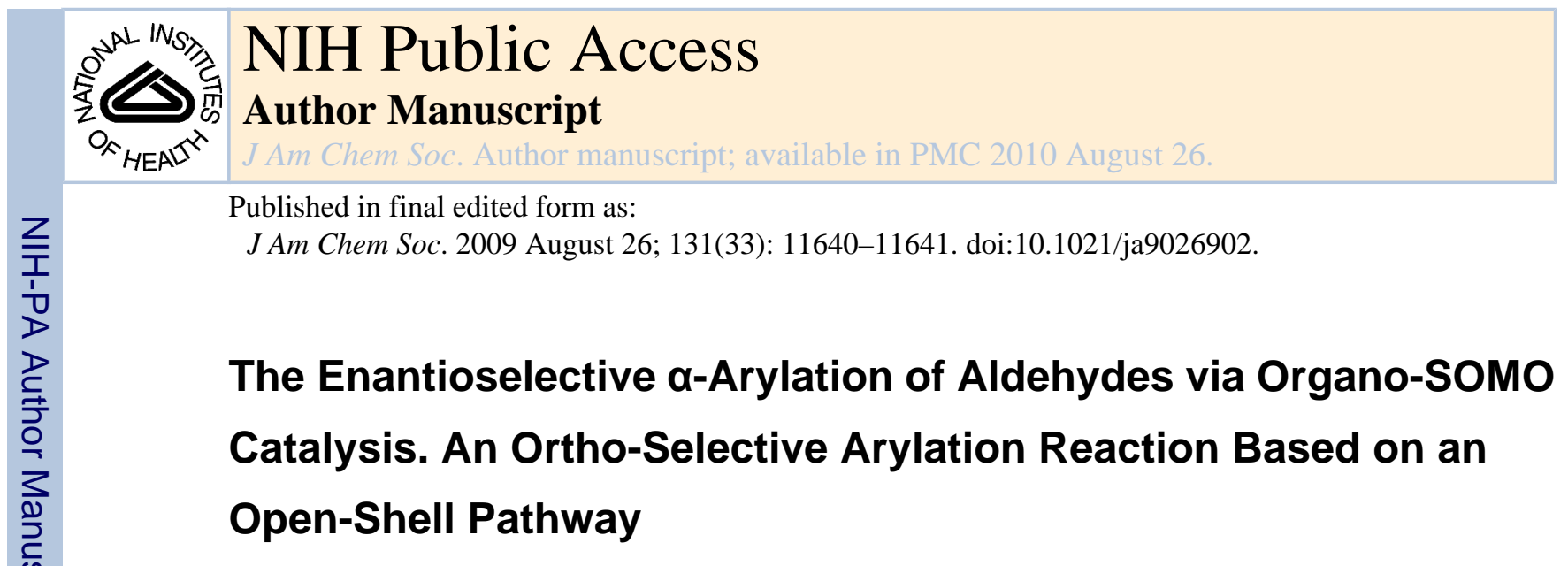

\author{
Jay C. Conrad, Jongrock Kong, Brian N. Laforteza, and David W. C. MacMillan \\ Merck Center for Catalysis at Princeton University, Princeton, New Jersey 08544, \\ dmacmill@princeton.edu
}

\begin{abstract}

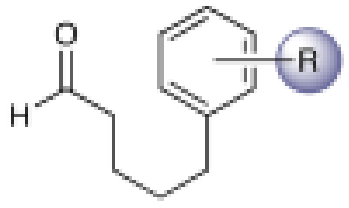

aldehyde

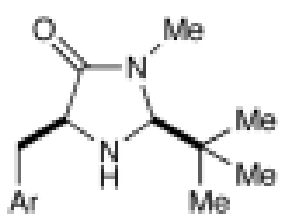

amine catalyst

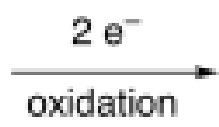

The intramolecular $\alpha$-arylation of aldehydes has been accomplished using singly occupied molecular orbital (SOMO) catalysis. Selective oxidation of chiral enamines (formed by the condensation of an aldehyde and a secondary amine catalyst) leads to the formation of a $3 \pi$-electron radical species. These chiral SOMO-activated radical cations undergo enantioselective reaction with an array of pendent electron-rich aromatics and heterocycles thus efficiently providing cyclic $\alpha$-aryl aldehyde products ( 10 examples: $\geq 70 \%$ yield and $\geq 90 \%$ ee). In accordance with our radical mechanism, when there is a choice between arylation at the ortho or para position of anisole substrates, we find that arylation proceeds selectively at the ortho position.
\end{abstract}

Driven primarily by advances in transition metal chemistry, the catalytic union of enolates with aryl halides has become a mainstay transformation in asymmetric synthesis. ${ }^{1}$ In particular, the pioneering work of Buchwald and Hartwig has provided a number of enantioselective enolate $\alpha$-arylations that allow the catalytic formation of $\alpha$-stereogenic carbonyls that are nonenolizable (e.g. quaternary carbon centers) ${ }^{2-4}$ Recently, our laboratory introduced a new mode of organocatalytic activation, termed SOMO catalysis, that is founded upon the mechanistic hypothesis that a $3 \pi$-radical cation species (e.g. 1) can readily participate in a range of unique asymmetric bond constructions. ${ }^{5}$ As part of these studies, we documented the first direct and enantioselective $\alpha$-allylic alkylation, ${ }^{5 \mathrm{~b}} \alpha$-enolation, ${ }^{5 \mathrm{c}}$ and $\alpha$-vinylation ${ }^{5 \mathrm{~d}}$ of aldehydes, three protocols that were not previously known. Continuing this theme, we recently questioned whether the $\alpha$-arylation of aldehydes might also be accomplished via SOMO catalysis to build and maintain enantioenriched benzylic stereocenters (eq 1). Herein, we describe the

Correspondence to: David W. C. MacMillan.

Supporting Information Available. Experimental procedures and spectral data are provided (x pages, PDF). 
enantioselective intramolecular aldehyde $\alpha$-arylation, a transformation that allows the highly efficient production of enolizable $\alpha$-formyl- $\alpha$-aryl substrates without post-reaction epimerization or quaternary carbon formation. ${ }^{6}$ Moreover, this protocol is highly orthoselective using 1,3-disubstituted aryl rings, a finding that is consistent with a radical based (open shell) arylation mechanism. ${ }^{7}$

Enantioselective $\alpha$-Arylation of Aldehydes via SOMO Catalysis (eq 1)
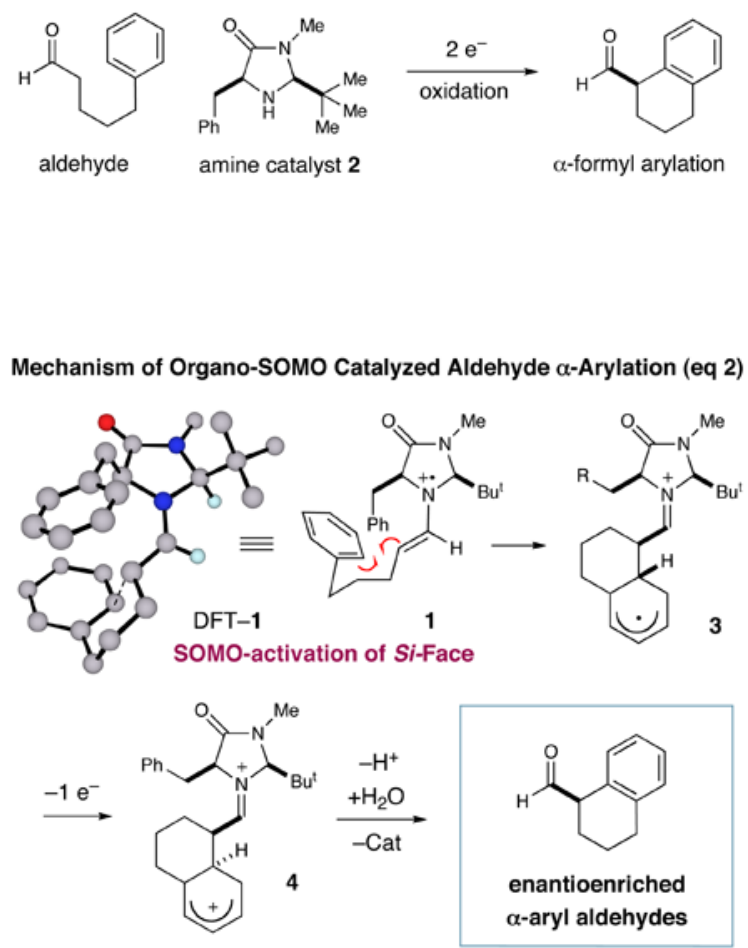

\section{Design Plan}

In accord with our previous SOMO-catalysis studies, ${ }^{5}$ we reasoned that exposure of an aryltethered aldehyde to amine catalyst $\mathbf{2}$ in the presence of a suitable oxidant would render the radical-cation 1 (DFT-1). Rapid and enantioselective addition of the pendent aryl ring system to the $3 \pi$-electron species would then provide a bicyclic radical cation 3 that upon further oxidation would generate a dienyl cation 4 (eq 2). At this stage, we presumed that rearomatization via proton loss would be facile while hydrolysis of the iminium species would reconstitute the catalyst and deliver the desired formyl $\alpha$-arylation product. Given the predilection of electron-rich aryl rings to undergo ortho-selective addition to radical cations, 7 we presumed that high levels of regiochemical control should be possible if a traditional SOMO catalysis mechanism is operative. Moreover, we presumed that the activated radical DFT -1 would position the $3 \pi$-electron system away from the bulky tert-butyl group, while the benzyl group on the imidazolidinone framework effectively shields the $R e$-face leaving the $S i$-face open to enantioselective arylation.

Our intramolecular aldehyde $\alpha$-arylation was first evaluated using 3-anisole pentanaldehyde, catalyst $\mathbf{2}$ or $\mathbf{5}$, and a selection of oxidants (Table 1). Initial experiments with ceric ammonium nitrate $(\mathrm{CAN})$ revealed that the proposed transformation was possible, however reaction 
efficiency and enantioselectivity levels were found to be moderate. Not satisfied with the general utility of these reaction conditions, we sought to find a superior catalyst/oxidant combination. As revealed in Table 1 , use of $\left[\mathrm{Fe}(\mathrm{phen})_{3}\right] \cdot\left(\mathrm{PF}_{6}\right)_{3}$ as a single electron oxidant allowed for a significant increase in enantiocontrol while application of a recently designed imidazolidinone catalyst 5 enabled optimal reaction efficiency and a further increase in stereocontrol (with $20 \mathrm{~mol} \%$ catalyst). High-throughput experiments using a robotic platform (see Supporting Information) revealed that tightly controlled quantities of additives such as pivalic acid and water could also be employed to boost efficiency (entries 3-6). Given the notable selectivities achieved with both amines $\mathbf{2}$ and $\mathbf{5}$, we selected both catalysts for further exploration.

As highlighted in Table 2, a wide array of aryl and heteroaryl ring systems readily participate as SOMOphiles in this intramolecular $\alpha$-aldehyde coupling. For example, anisole, naphthylene, indole, pyrrole, thiophene and furan ring systems all function well ${ }^{9}$ in this new enantioselective ring forming reaction. Moreover, aldehydes that incorporate nitrogen, oxygen or carbonyl containing tethers worked well in this protocol (entries 2, 4, and 10, catalyst 5: 93-98\% ee). As is clearly shown in Table 2, the imidazolidinone catalyst $\mathbf{5}$ is far superior to amine $\mathbf{2}$ with respect to enantioselectivity and cyclization efficiency. It is also important to note that these electron-rich aryl ring systems are completely tolerant to the mild oxidative conditions employed in this study. Furthermore, substrates that have the potential for aromatic substitution at two regiochemical positions (ortho or para) were in fact highly selective for orthofunctionalization (entries $1-5$, ortho-substitution only). ${ }^{10}$ Such regioselectivity provides further evidence that a radical-mediated addition pathway is operative, as proposed in equation 2. It should be noted that a cationic Friedel-Crafts mechanism was recently proposed for a similar arylation reaction using our imidazolidinone catalyst $2 ; 8$ a mechanistic interpretation based on the exclusive observation of para-selective products. However, the authors have subsequently issued a correction ${ }^{8 b}$ that several of their adducts were in fact formed with orthoselectivity (Table 3, entries 1 and 3). As such, given (i) our previous mechanistic studies into SOMO-activation, wherein an open-shell pathway was definitively shown using a radicalclock probe, ${ }^{5 \mathrm{a}}$ and (ii) the observation of only ortho-selective products using 1,3-disubstituted aryl rings, we presume that a radical cyclization is operative for the substrates described in this manuscript. ${ }^{9}, 10$

\section{Sequential Organocatalytic Conjugate Reduction-SOMO Arylation}

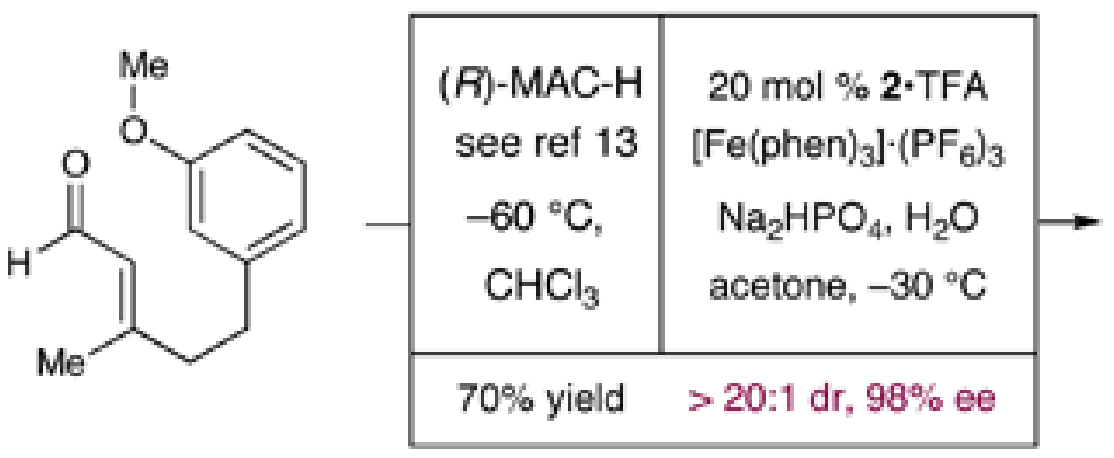<smiles>COc1cccc2c1[C@H](C=O)[C@H](C)CC2</smiles> 


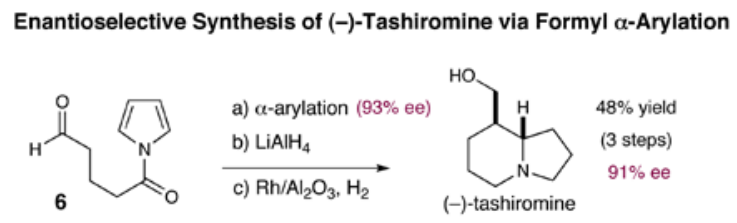

We have been able to combine our novel SOMO a-arylation reaction into a two-step organocatalytic sequence that first involves a Hantzsch ester hydride (HEH) reduction ${ }^{11}$ of an anisole tethered $\alpha, \beta$-unsaturated aldehyde followed by the protocol described herein. As shown in equation 4 , this sequence provides the resulting bicyclic ring system as a single (anti) diastereomer in $70 \%$ yield and $98 \%$ ee.

We have also been able to apply this new carbonyl $\alpha$-arylation technology towards the total synthesis of (-)-tashiromine. ${ }^{12}$ As outlined in equation 5, exposure of the pyrrole amide tethered aldehyde 6 to this new catalytic procedure provides the desired [6,5]-bicyclic ring system in $72 \%$ yield and in $93 \%$ ee. The resulting amide was then reduced in the presence of $\mathrm{AlCl}_{3}$ with $\mathrm{LiAlH}_{4}$ in $83 \%$ yield prior to exhaustive pyrrole hydrogenation using catalytic Rh/ $\mathrm{Al}_{2} \mathrm{O}_{3}$ and $\mathrm{HOCH}\left(\mathrm{CF}_{3}\right)_{2}$ to provide (-)-tashiromine in $81 \%$ yield and $4: 1$ diastereoselectivity.

\section{Supplementary Material}

Refer to Web version on PubMed Central for supplementary material.

\section{Acknowledgments}

Financial support was provided by NIHGMS (R01 GM078201-01-01) and kind gifts from Merck. JCC thanks NSERC for a postdoctoral fellowship.

\section{References}

1. a) Muratake H, Nakai H. Tetrahedron Lett 1999;40:2355. b) García-Fortanet J, Buchwald SL. Angew Chem Int Ed 2008;47:8108. c) Vo GD, Hartwig JF. Angew Chem Int Ed 2008;47:2127.

2. a) Liao X, Weng Z, Hartwig JF. J Am Chem Soc 2008;130:195. [PubMed: 18076166] b) GarcíaFortanet J, Buchwald SL. Angew Chem Int Ed 2008;47:8108.

3. This racemization has been exploited for dynamic kinetic resolutions: Xie JH, Zhou ZT, Kong WL, Zhou QL. J Am Chem Soc 2007;129:1868. [PubMed: 17263537]

4. With quinone Michael acceptors: Alemán J, Cabrera S, Maerten E, Overgaard J, Jørgensen KA. Angew Chem Int Ed 2007;46:5520.

5. a) Beeson TD, Mastracchio A, Hong J-B, Ashton K, MacMillan DWC. Science 2007;316:582. [PubMed: 17395791] b) Jang H-Y, Hong J-B, MacMillan DWC. J Am Chem Soc 2007;129:7004. [PubMed: 17497866] c) Kim H, MacMillan DWC. J Am Chem Soc 2008;130:398. [PubMed: 18095690] d) Graham TH, Jones CM, Jui NT, MacMillan DWC. J Am Chem Soc 2008;130:16494. [PubMed: 19049447]

6. Details of this work were described at the ACS Centennial Symposium MacMillan, David W. C. Abstracts of Papers, 236th ACS National Meeting, Philadelphia, PA, United States, August 17-21, 2008 (2008), ORGN-545. CODEN: 69KXQ2 AN 2008:954577 CAPLUS

7. For ortho-selective radical additions to anisole see: Tiecco M, Testaferri L. Abramovitch RA. Reactive Intermediates 3Plenum PressNew York1983;:61.

8. Nicolaou KC, Reingruber R, Sarlah D, Bräse S. J Am Chem Soc 2009;131:2086. [PubMed: 19173649] (b) A correction for ref 8a was submitted on the same day as the original version of this manuscript (which also noted the mischaracterizations of ref 8a): J. Am. Chem. Soc. 2009, 131, 6640. While the 
authors of $8 \mathrm{a}$ and $8 \mathrm{~b}$ did not provide experimental verification of the reassigned structures, our submission did so, see Supporting Information.

9. It should be noted that Nicolaou and co-workers observed para-selectivity with highly electron-rich 1,3,4-trisubstituted aryl rings (see ref $8 \mathrm{a}$ ).

10. An open shell, radical pathway is also consistent with the successful addition of highly electrondeficient styrenes to radical cations such as 1 (see ref $5 \mathrm{~d}$ ).

11. a) Ouellet SG, Walji A, MacMillan DWC. Acc Chem Res 2007;40:1327. [PubMed: 18085748] b)

Ouellet SG, Tuttle JB, MacMillan DWC. J Am Chem Soc 2005;127:32. [PubMed: 15631434]

12. Banwell MG, Beck DAS, Smith JA. Org Biomol Chem 2004;2:157. [PubMed: 14737636] 
Table 1

Development of Organo-SOMO Aldehyde $\alpha$-Arylation.<smiles>COc1cccc(CCCCC=O)c1</smiles><smiles>CN1C(=O)[C@@H](C[Al])N[C@H]1C(C)(C)C</smiles>

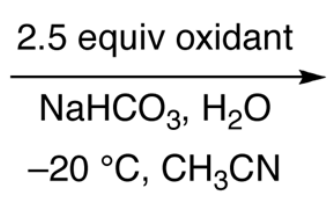<smiles>COc1cccc2c1[C@H](C=O)CCC2</smiles>
aryl aldehyde amine catalyst $\alpha$-aryl aldehyde

\begin{tabular}{|c|c|c|c|c|}
\hline entry & oxidant & catalyst (Ar) \% & yield $^{a}$ & $\% \mathrm{ee}^{b}$ \\
\hline 1 & CAN & $\mathrm{Ph}, 2(30 \mathrm{~mol} \%)$ & 63 & 84 \\
\hline 2 & {$\left[\mathrm{Fe}(\text { phen })_{3}\right] \cdot\left(\mathrm{PF}_{6}\right)_{3}$} & $\mathrm{Ph}, 2(30 \mathrm{~mol} \%)$ & 60 & 92 \\
\hline $3^{c}$ & $\left.\left[\mathrm{Fe}(\text { phen })_{3}\right] \cdot \mathrm{PF}_{6}\right)_{3}$ & $\mathrm{Ph}, 2(30 \mathrm{~mol} \%)$ & 85 & 92 \\
\hline $4^{c}$ & {$\left[\mathrm{Fe}(\text { phen })_{3}\right] \cdot\left(\mathrm{PF}_{6}\right)_{3}$} & $\mathrm{Ph}, 2(20 \mathrm{~mol} \%)$ & 61 & 90 \\
\hline $5^{c}$ & {$\left[\mathrm{Fe}(\text { phen })_{3}\right] \cdot\left(\mathrm{PF}_{6}\right)_{3}$} & 1-Naphth, 5 (20 mol \%) & 70 & 97 \\
\hline $6^{c, d}$ & {$\left[\mathrm{Fe}(\text { phen })_{3}\right] \cdot\left(\mathrm{PF}_{6}\right)_{3}$} & 1-Naphth, 5 (20 mol \%) & 80 & 98 \\
\hline
\end{tabular}

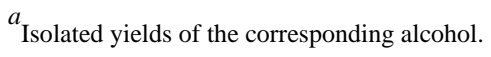

${ }^{b}$ Enantiomeric excess determined by chiral HPLC.

$c_{30 \mathrm{~mol} \% \text { pivalic acid. }}$

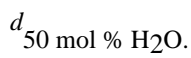



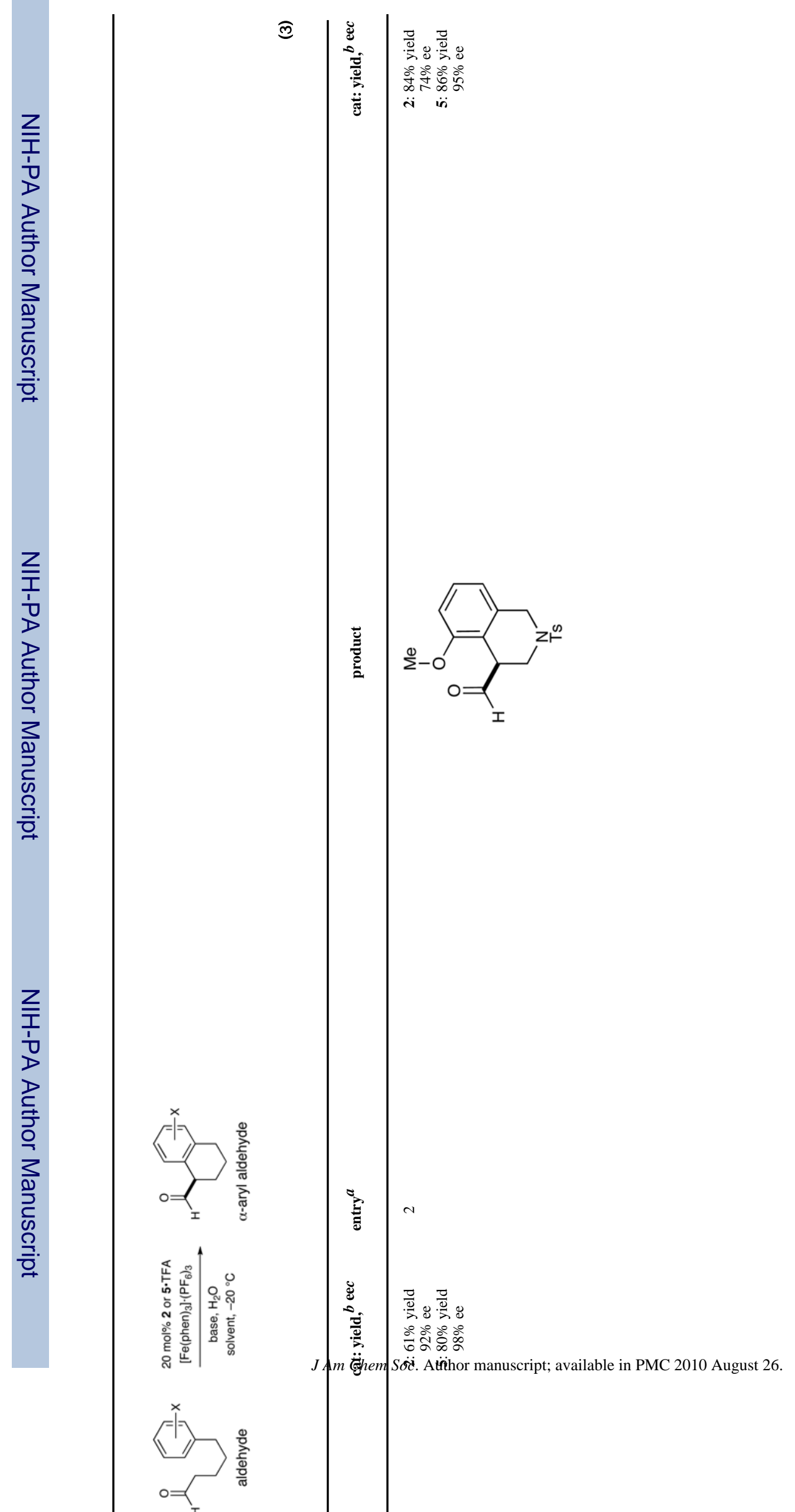


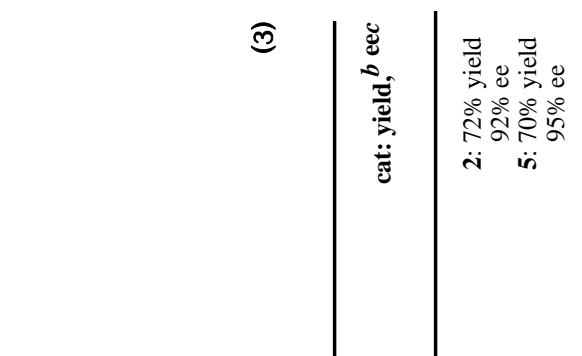

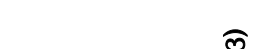

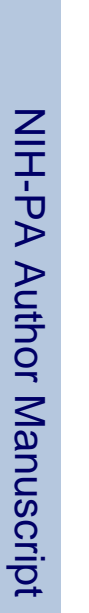

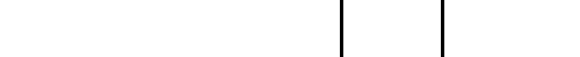

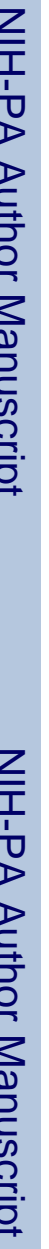

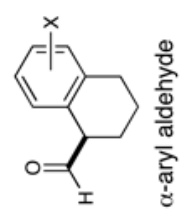

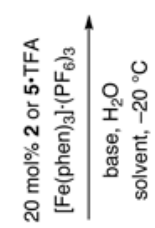

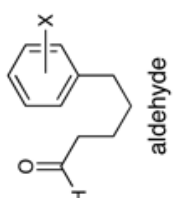




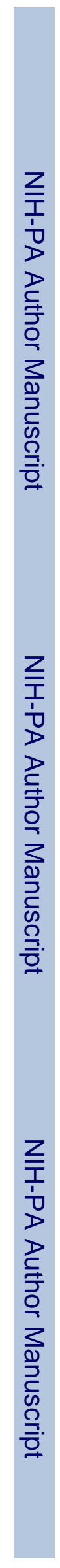

Conrad et al.

్

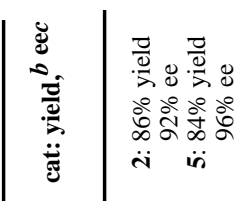

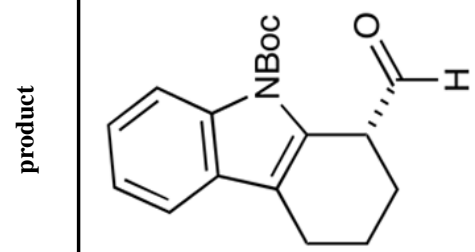

$\underbrace{\frac{1}{20}}_{I}$

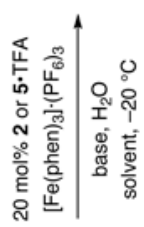

$\stackrel{0}{E}$

6

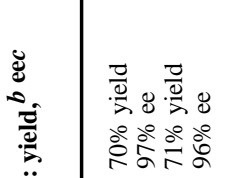

苞 An Ctiem
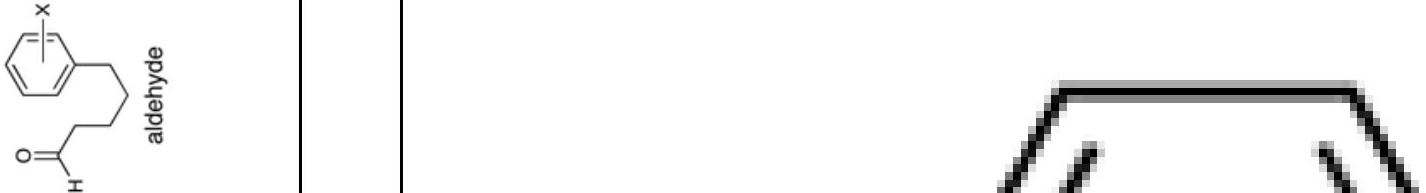
ิ్

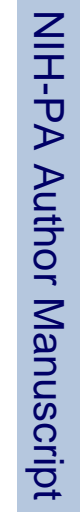

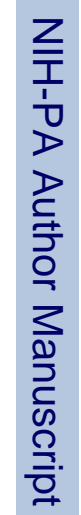

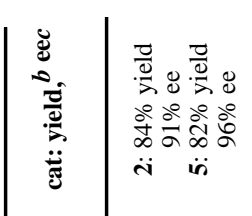




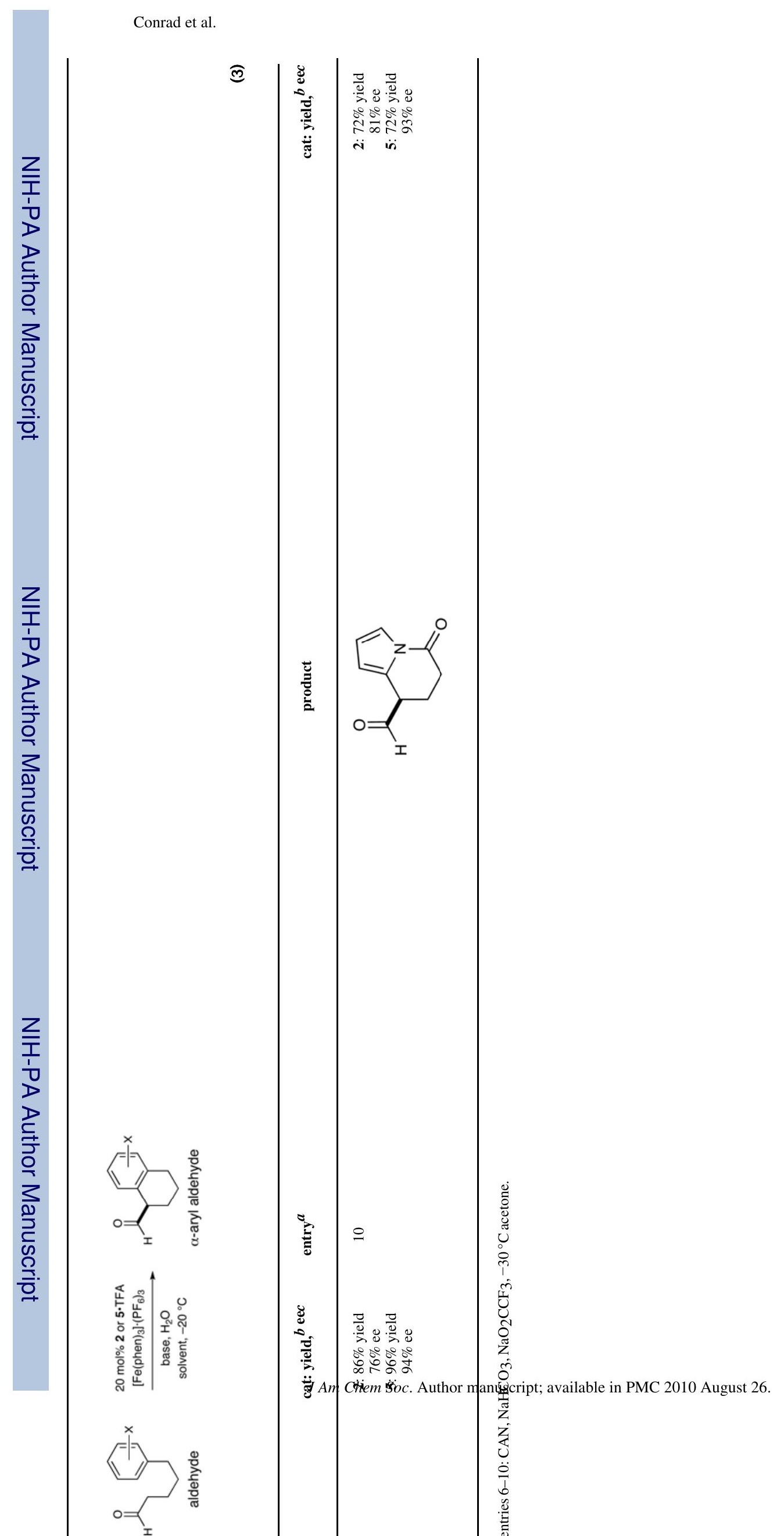

Page 11 